\title{
A SELF-POWERED WIRELESS SENSOR NETWORK FOR DYNAMIC MANAGEMENT OF QUEUES AT TRAFFIC LIGHTS
}

\author{
Mario Collotta ${ }^{1}$, Mario Denaro $^{2}$, Gianfranco Scatà $^{3}$, Antonio Messineo $^{4}$, \\ Giuseppina Nicolosi ${ }^{5}$
}

\author{
Faculty of Engineering and Architecture \\ Kore University of Enna, Cittadella Universitaria, 94100 Enna, Italy \\ E-mails: ${ }^{1}$ mario.collotta@unikore.it; ${ }^{2}$ mario.denaro@unikore.it; ${ }^{3}$ gianfranco.scata@unikore.it; \\ ${ }^{4}$ antonio.messineo@unikore.it; ${ }^{5}$ giuseppina.nicolosi@unikore.it
}

\begin{abstract}
The dynamic management of traffic light cycles is a really interesting research issue considering modern technologies, which can be used in order to optimise road junctions and then improve living conditions of the roads. Wireless sensor networks represent the most suitable technology, as they are easy to deploy and manage. The data relating to road traffic flows can be detected by the sensor network and then processed through the innovative approach, proposed in this work, in order to determine the right green times at traffic lights. Although wireless sensor networks are characterized by very low consumption devices, the continuous information transmission reduces the life cycle of the whole network. To this end, the proposed architecture provides a technique to power the sensor nodes based on piezoelectric materials, which allow producing potential energy taking advantage of the vibration produced by the passage of vehicles on the road.
\end{abstract}

Keywords: Wireless Sensor Networks; Piezoelectric; Traffic Lights; Intelligent Transportation Systems

\section{Introduction}

During last years, research in the field of Intelligent Transportation Systems (ITS) is focusing on new techniques and technologies to be used in efficient and automated transportation systems aimed to save energy. The issue of self-powered ITS control systems has become particularly important, especially in areas, which use wireless technologies, where there are neither wired connections nor power-lines. Therefore the main aim is to find new solutions in order to power devices through the cars' movement detected by the same devices placed on the road. Most of traffic lights are characterized by "fixed cycles". As a consequence, green times are not balanced because a static management mechanism does not take into account the length of the queues. The waiting times, therefore, increase proportionally with the increase of the queue length. WSN are perfect for vehicular traffic flows detection [1] thanks to their ease of use and management. Moreover, they can be used anywhere regardless of the environmental conditions and support a high degree of energy saving. Energy harvesting applications have been developed in order to produce electric energy from vehicular, train or pedestrian traffic or from environmental vibration in general. However, there are no applications, which can develop energy from roads usable by themselves (by ITS systems). Several kinds of energy sources have been investigated by researchers. The vibration sources are the most ubiquitous and can be found everywhere [2-3]. The mechanical energy can substitute solar energy, sometimes with more potentiality. In both cases, the objective regards a greater amount of energy generated as efficiently and economically as possible. Energy harvesting, using smart materials such as piezoelectric, has been studied and developed in different scenarios [4]. Recently, an effective way for the electricity production is represented by the recovery of mechanical energy produced by vibrations. This can be obtained through the use of piezoelectric materials, taking advantage from piezoelectric effect that consists of the generation of an electric field produced by a mechanical deformation of the same piezoelectric material. During last years, this technology has been applied in several fields, like self-powered wireless sensor networks for data acquisition and processing [5]. This paper presents an innovative technique for the dynamic management of traffic light cycles based on realtime information detected by self-powered sensor nodes placed along road sections. The main aim of the approach is to reduce the waiting time in traffic light queues. The paper is organized as follow: section 2 proposes main literature related works while section 3 describes the network architecture. Section 4 proposes the experimental test-bed showing, at the same time, obtained results. Section 5 summarizes the paper and reports conclusion and possible future works. 


\section{Related Works}

The work here proposed shows a dynamic management technique of traffic light cycles based on information gathered by self-powered sensors. To better understand the state of art, this section will be divided into two sub-parts. The first part will show the main techniques of road traffic information processing through the wireless sensor networks, while the second will show the main literature works dealing with energy harvesting.

\subsection{Wireless Sensor Networks for road traffic management}

WSNs can be used in several fields like home automation [6-7], health [8], biometric systems [9], industry [10-11] and road monitoring. Considering the increasing use of this technology, the researchers aim to develop architectures and techniques in order to obtain a high-performance use of WSNs in several and complex fields like Intelligent Transportation Systems. In [12], the traffic flows scheduling is managed using an algorithm called TRED (Traffic Random Early Detection) based on the RED algorithm, the most common Active Queue Management (AQM) algorithm [13] used to manage congestions in computer networks. The algorithm evaluates the saturation of a road, based on some measured information like the average length of vehicles, and forwards the cars along alternative ways in case of congestion detected. In [14], the authors describe an architecture in which sensor nodes detect road information and send them to the nearest Intersection Control Agent (ICA), which determines the flow model of the intersection depending on sensors data. In [15] and [16] the authors proposed an adaptive traffic light control algorithm that adjusts both the sequence and length of traffic lights in accordance with the real time traffic detected. This algorithm considers a number of traffic factors such as traffic volume, waiting time, vehicle density, etc., to determine green light sequence and the optimal green light duration. In [17] the authors propose an algorithm for vehicle detection, using magneto resistive sensors, in order to control road traffic. The system developed is able to detect vehicles and then to calculate the appropriate duration of traffic light cycles. In [18] the authors propose a fuzzy method in order to dynamically adjust the delay of green light according to the real-time vehicle flows detected by a wireless sensor network. The system mainly consists of four wireless sensor nodes (SN) and a traffic light control node $(\mathrm{CN})$. Each sensor node contains one magnetic sensor, which is used to detect vehicles by measuring the change in the Earth's magnetic field caused by the presence of a vehicle near the sensor.

\subsection{Energy harvesting in ITS systems}

Energy demand in recent years is increasing: the reserves of fossil fuels are running out. Moreover the increasing cost of energy and related environmental problems have led researchers to investigate new energy sources [19]. Some innovative mechanisms have been studied to reduce greenhouse gas emissions by increasing energy efficiency of existing systems. Concrete solutions have been introduced for saving and energy recovery in both civil and industrial environments [20-21]. Some works discuss the energy harvesting using piezoelectric materials. Today, the energy recovery techniques using environmental mechanical vibrations are very interesting research issues. Recently, in the station of Shibuya (Tokyo), was installed an energy collection system that uses a piezoelectric transducer [22], under a proposal of the East Japan Railway Company. Also the society "Innowatech", in recent years, has proposed some applications based on piezoelectric stack transducer for the energy production taking advantage of vehicular traffic [22]. Some researchers have recently shown a mechanical device for energy harvesting using piezoelectric bimorph bender transducer, which can recover energy from road, pedestrian and rail traffic. The innovation of this technique is represented by the use of piezoelectric bender devices and by an innovative configuration to transfer mechanical vibrations of the main box to the piezoelectric transducer [23].

\section{System Architecture and Requirements}

Intelligent Transportation Systems (ITS) are characterized by several and smart embedded devices in order to support road monitoring and decision making. The proposed architecture consists, as said, in a self-powered wireless sensor network able to measure road traffic flows and determine the traffic-light cycles based on the queue length near the road junction. Main devices used are: 
- $\quad$ Self-powered Reduced Function Devices (RFDs), each one with a magnetic sensor in order to evaluate the earth's magnetic field distortion caused by the presence of cars;

- Self-powered Full Function Devices (FFDs), which gather information received by their RFDs and forward them to the First Pan Coordinator (FPC);

- Self-powered First Pan Coordinator. It is the network coordinator. Its main aim is to gather all information in order to make decisions for a better management of the network.

In the proposed architecture, the FPC is characterized by three modules:

- a data gathering module;

- $\quad$ an IEEE 802.15.4 [24] module;

- a decision making module, which main aim is to dynamically adjust traffic light cycles based on information detected by the whole network.

The proposed architecture is really complex and this is the reason why, this section has been divided into sub-sections. In sub-section 3.1 we will analyse the dynamic traffic light manager explaining how the proposed algorithm works; sub-section 3.2 will show self-power technologies used for IEEE 802.15.4 sensors while in last sub-section will be explained the electrical circuit model and the battery charging system.

\subsection{Dynamic traffic light manager}

In order to explain our approach, let we consider a general road intersection (Figure 1), where $\mathrm{R}_{\mathrm{A}}$ is the road section containing traffic lights 2 and 4 and $R_{B}$ is the road containing traffic lights 1 and 3 .

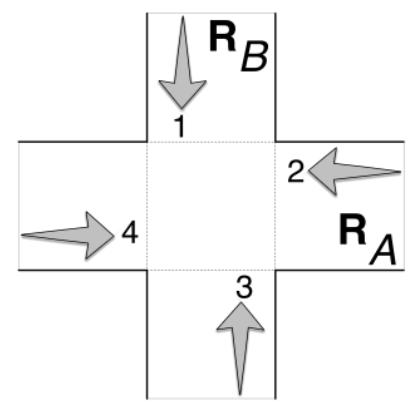

Figure 1. Road intersection

Our goal is to realize a decision making algorithm in order to dynamically evaluate traffic light cycles based on the queue length. Figure 2 shows a road section of length $\boldsymbol{L}$ subdivided into smaller subsections, each one of length $\boldsymbol{l}_{\boldsymbol{i}}$. Each subsection $\boldsymbol{l}_{\boldsymbol{i}}$ is monitored by a node in order to detect vehicles presence through a magnetic sensor, which measures the earth's magnetic field distortion due to the presence of ferrous objects (vehicles) [25]. Each node communicates with other nodes and the FPC according to the IEEE 802.15.4 [24] standard protocol. The algorithm evaluates the traffic light cycles considering three fundamental parameters

- $\quad$ the road section length $(\boldsymbol{L})$ to be monitored;

- $\quad$ the sub-sections length $\left(\boldsymbol{l}_{\boldsymbol{i}}\right)$;

- $\quad$ approximate crossing speed $(v)$.

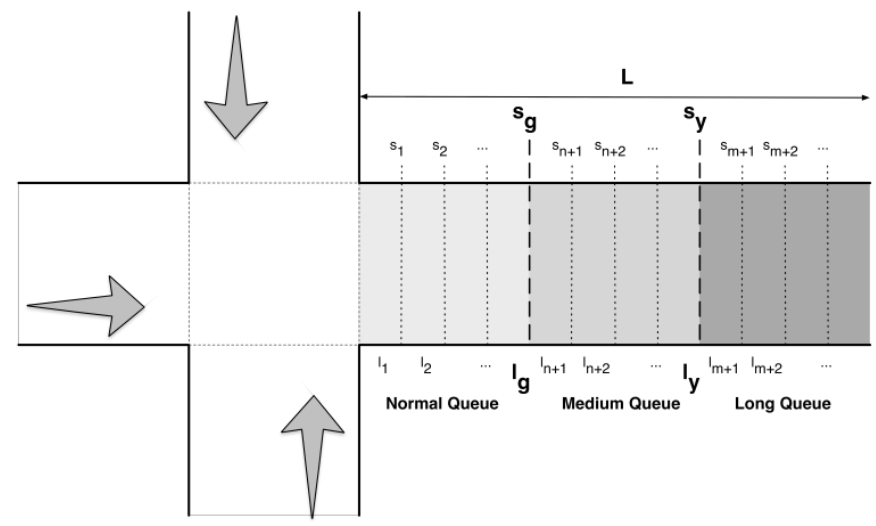

Figure 2. Monitored road section by the wireless sensor network 
The average length of a car is between 3.5 and 5 meters even if there are vehicles both longer and shorter. As a consequence, the length of each subsection $\left(\boldsymbol{I}_{\boldsymbol{i}}\right)$ can be between 4 and 8 meters. According to IEEE 802.15.4, the RFD nodes can be placed every 12 meters while the FFD nodes every 60 meters, while the FPC can be placed at the end of the road near the traffic light. In a small city, the average speed ( $v$ ), of a vehicle crossing a traffic light intersection is, at about, $15 \mathrm{Km} / \mathrm{h}$. Let consider $\boldsymbol{I}_{\boldsymbol{g}}$ the maximum value within, which a traffic light queue is considered normal. $\boldsymbol{L}_{y}$ is the maximum value within, which the queue can be considered medium. Above this value, the queue is considered long. The number of subsections is calculated as $L / \boldsymbol{l}_{\boldsymbol{i}}$ while the $\boldsymbol{I}_{\boldsymbol{g}}$ value can be determined by the following relation (1):

$l_{g}=\frac{L}{l_{i}}+1$.

The $\boldsymbol{l}_{\boldsymbol{y}}$ value, therefore, is calculated through equation (2):

$l_{y}=\frac{L}{l_{i}}-l_{\mathrm{g}}$.

In case of vehicles detection in a section $\boldsymbol{I}_{\boldsymbol{i}}<\boldsymbol{I}_{\boldsymbol{g}}$ the traffic light works under standard conditions, with period $\boldsymbol{T}_{\boldsymbol{i}}$, green time $\boldsymbol{t}_{\boldsymbol{g}}$ and yellow time $\boldsymbol{t}_{\boldsymbol{y}}$. In case of vehicles detection, in a section $\boldsymbol{I}_{\boldsymbol{g}}<\boldsymbol{l}_{\boldsymbol{i}}<\boldsymbol{I}_{\boldsymbol{y}}$, the estimated queue length is considered medium. Instead, $\boldsymbol{l}_{\boldsymbol{i}}>\boldsymbol{l}_{\boldsymbol{y}}$, is the condition of long queue.

The crossing time of a single subsection can be easily calculated through equation (3):

$t_{i}=\frac{l_{i}}{v}$,

where $\boldsymbol{I}_{\boldsymbol{i}}$ represents the $i$-th section length while $\boldsymbol{v}$ is the approximate crossing speed of the considered road section. In case of medium or long queue, the green time is re-calculated taking into account the estimated queue length. Considering the subdivision of the sub-sections shown on Figure 3, the standard green time $\boldsymbol{t}_{\boldsymbol{g}}$ and the real-time green time $\boldsymbol{t}_{\text {grt }}$ can be calculated through the following relations (4).



Figure 3. Sections subdivision

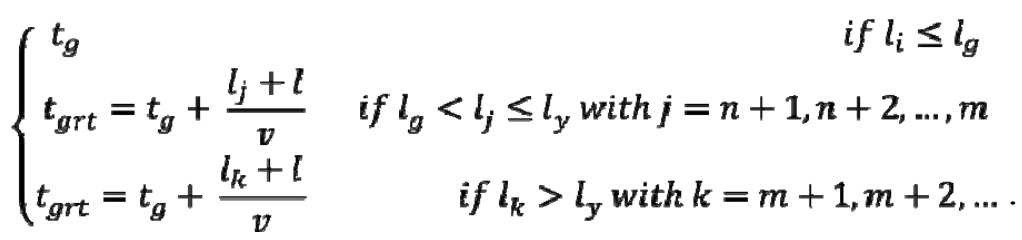

The terms $\left(l_{k}+l\right) / v$ and $\left(l_{j}+l\right) / v$ represent, each one, ratios between space and speed. In other words, they represent values of time to be added to the standard green time $\boldsymbol{t}_{\boldsymbol{g}}$ in case of increasing queue in order to slightly increase the total green time and to allow the real-time management of the traffic light according to the queue behaviour. Clearly, the road with the longest green time has higher priority. Each traffic light independently calculates, during each period, its green time in order to determine its priority level according to the following relations (5):

$$
\begin{array}{lcl}
p_{i}=t_{g} & \text { if } l_{i}<l_{g} & \text { with } i=1,2,3,4 \\
p_{i}=t_{g r t} & \text { if } l_{i}>l_{g} & \text { with } i=1,2,3,4
\end{array}
$$


where $\boldsymbol{p}_{\boldsymbol{i}}$ is the priority of the $i$-th traffic light. At the end of the period $\mathbf{T}_{\boldsymbol{i}}$, the algorithm determines the road with the highest priority determining, which traffic light needs more green time.

The priority of each road is calculated through equation (6) and (7), as the average of the individual priorities calculated by each traffic light adding a $\Delta$ value (which can be positive or negative). This $\Delta$ value is then divided by two in order to avoid that the priority, acquired by a road, can be too high (excessive green times).

$P_{R_{A}}=\frac{\left(p_{1}+p_{3}\right)}{2}+\frac{\Delta_{A}}{2}$

$P_{R_{B}}=\frac{\left(p_{2}+p_{4}\right)}{2}+\frac{\Delta_{B}}{2}$

\subsection{Self-power technologies used for IEEE 802.15.4 sensors}

The adopted configuration, used to supply power sensor nodes, is characterized by a bimorph piezoelectric transducer rectangular clamped to an extreme [26]. This converter consists of two piezoelectric outside (active layers) and a thin metal plate in the middle section (passive layer). The passive layer performs both the connection between the two active layers, and the distancing of the same from the neutral axis, resulting in increased deformations and electric potential [26]. The converter is made of lead zirconate titanium (PZT-5A), a material with a high efficiency and flexibility in the charge production (Table 1).

Table 1. Electromechanical Parameters

\begin{tabular}{c|c|c|c}
\hline \hline Symbol & Quantity & Value & Units \\
\hline \hline$l p$ & Piezo length & 40,0 & $\mathrm{~mm}$ \\
\hline$w p$ & Piezo width & 16,0 & $\mathrm{~mm}$ \\
\hline$t p$ & Piezo thickness & 0,80 & $\mathrm{~mm}$ \\
\hline$t p u$ & Upper patch thickness & 0,36 & $\mathrm{~mm}$ \\
\hline$t p l$ & Lower patch thickness & 0,36 & $\mathrm{~mm}$ \\
\hline$t s p$ & Steel plate thickness & 0,08 & $\mathrm{~kg} / \mathrm{m}^{3}$ \\
\hline$\rho p$ & Piezo density & $7.750,0$ & $\mathrm{~kg} / \mathrm{m}^{3}$ \\
\hline$\rho s$ & Steel density & $7.850,0$ & $\mathrm{~Pa}$ \\
\hline $\mathrm{Ep}$ & PiezoYoung's modulus & 6,61010 & $\mathrm{~Pa}$ \\
\hline $\mathrm{Es}$ & Steel Young's modulus & 20,51010 & $\mathrm{~N} / \mathrm{mm}$ \\
\hline $\mathrm{K}$ & Elastic constant & 30,8 &
\end{tabular}

Two different polarization configurations have been analysed: parallel and serial. The first involves the construction of three electrical connections for the extraction of the converted charge, while the latter requires the adoption of two electrical connections. Figure 4 shows the constructive characteristics of the two configurations adopted, with the electric connections and the polarization directions [27].


a)



b)

Figure 4. Bimorph piezoelectric. a) Parallel configuration (left); b)serial configuration (right) 
The Energy Harvesting device will be installed within a road cavity in order to catch the external vibrations, caused by the passage of vehicles, in order to transform them in energy [28]. The system consists of a piezoelectric transducer bonded on the surface of the steel beam subjected to external impulse [29]. The beam undergoes a tensile stress and a compression producing, as a consequence, electrical potential [23] (Figure 5). This potential difference, generated at the extreme poles through an electrical circuit, is converted into electrical energy.


Figure 5. a) Road cavity; b) Detail of the Energy Harvesting installed inside the speed bump with cavity

\subsection{Model electrical circuit and battery charging system}

The energy obtained from the piezoelectric transducers, is not directly usable by electronic devices, because of random variations of power and voltage over the time. It is therefore necessary, a suitable circuit for the management of gathered power. The effort made to obtain efficient transducers, may be lost without the use of adapters able to convert signals of a few millivolts, or even less, without substantial losses. These circuits also consume energy and must be able to shut down when the source of energy is not enough to power the device in order to avoid unnecessary energy consumption. When the energy increases, the power management circuit must be able to automatically switch-on (self-starting). The simplicity of the circuital technology depends on the availability of space and energy. The general structure of the power manager circuit can be divided in three interfaces, as shown on Figure 6 a). The output voltage of a piezoelectric generator is generally characterized by a pseudo-periodic behaviour and assumes positive and negative values alternately. Therefore, it is always necessary to a rectifier circuit [30].

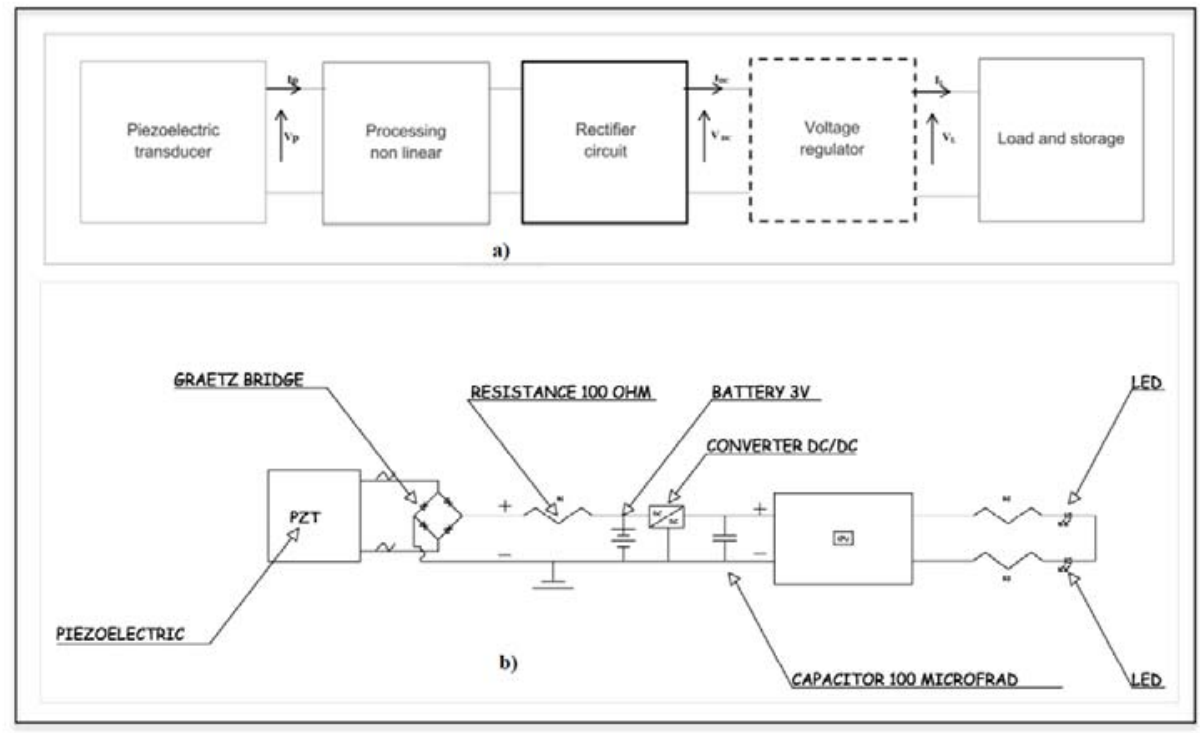

Figure 6. a) General structure of a power manager circuit [32];

b) Energy harvester simulation model including mechanical part and bridge diode rectifier power conditioning 
The circuit uses a small piezoelectric transducer in order to convert the mechanical vibration into an $\mathrm{AC}$ voltage source that is powered inside the bridge rectifier internal of the LTC3588. It can recover small vibrations and generate system power instead of using traditional batteries. The LTC3588-1 is a very low quiescent current power supply, specially designed for energy recovery applications or stepdown at low current. It can directly interface with a piezoelectric transducer or another AC power source, rectifying the voltage waveform and storing the energy recovered in an external storage capacitor. At the same time it dissipates the energy in excess through an internal derivation regulator, maintaining an output voltage regulated through a "buck" regulator with high efficiency. The full-wave bridge rectifier inside the LTC3588-1 is accessible from two differential inputs, PZ1 and PZ2, which rectify the AC inputs. The rectified output is stored in a capacitor to the VIN pin and can be used as an energy reserve for the "buck" converter. The low loss bridge rectifier has a total voltage drop of about $400 \mathrm{mV}$ with typical piezoelectric currents, which are normally equal to about $10 \mu \mathrm{A}$. This bridge is able to conduct currents up to $50 \mathrm{~mA}$. The "buck" regulator is enabled as soon as an enough voltage is available on VIN in order to produce a regulated output. The "buck" regulator uses an algorithm for hysteretic voltage in order to control the output through the internal feedback of the detection pin VOUT. The "buck" converter loads an output converter through an inductor to a value slightly higher than the regulation point. To this end, the current inductor is increased to $260 \mathrm{~mA}$ through an internal PMOS switch and then decreased to $0 \mathrm{~mA}$ through an internal NMOS switch. In this way, the energy is efficiently supplied to the output capacitor. This hysteretic method reduces losses associated with FET switching and holds low the output loads. In the switching process, the "buck" converter provides at least $100 \mathrm{~mA}$ of average load current. On Figure 6 b) it is graphically represented the realized circuit that manages the output AC current from the piezoelectric transducer [31]. The AC standard is the simplest form of power conditioning and directly connects a resistive load between the two electrodes of the considered transducer. The DC standard rectifies the output transducer, through a full bridge diode rectifier, and connects a resistive load in parallel with a storage capacitor at the output of the rectifier.

\section{Performance Evaluation}

To better understand how the proposed system has been evaluated, even this section has been divided into two sub-sections. The first one deals with the performance obtained using the dynamic traffic light manager compared to fixed traffic light cycles. In section 4.2 instead will be shown simulation results related to the piezoelectric device for sensors' charge.

\subsection{Traffic lights manager evaluation}

Our approach has been proved in a normal track crossroad. The simulations have been carried out both in case of fixed and dynamic traffic light cycle. Performance has been evaluated considering that up to 80 vehicles may approach each traffic light. In other words, up to 160 vehicles can be measured on each road. In both cases, the reference cycle was 60 seconds and measurements have been gathered for 60 cycles ( 1 hour). Figures 7 and 8 show the number of vehicles measured in Road A in case of Fixed Cycle and Dynamic Cycle respectively. The Figure 7 shows how, in case of fixed-cycle, sensors detect on average 23.08 vehicles/minute in transit. Of these, just 10.48 are disposed.

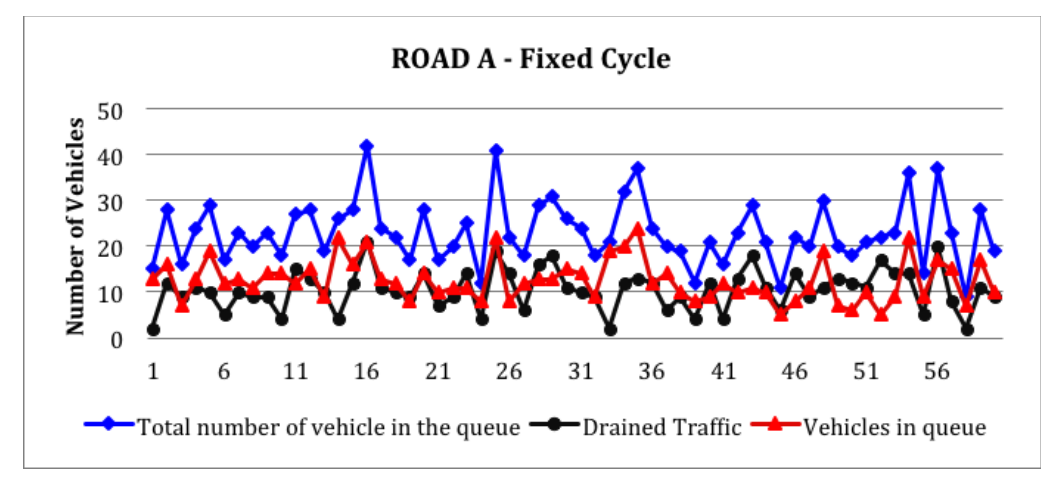

Figure 7. Road A - measures obtained with fixed-cycle 
On the contrary, the Figure 8 shows that, in case of dynamic-cycle, on average 20.98 vehicles/minute are disposed, about the $91 \%$ of total vehicles transited.

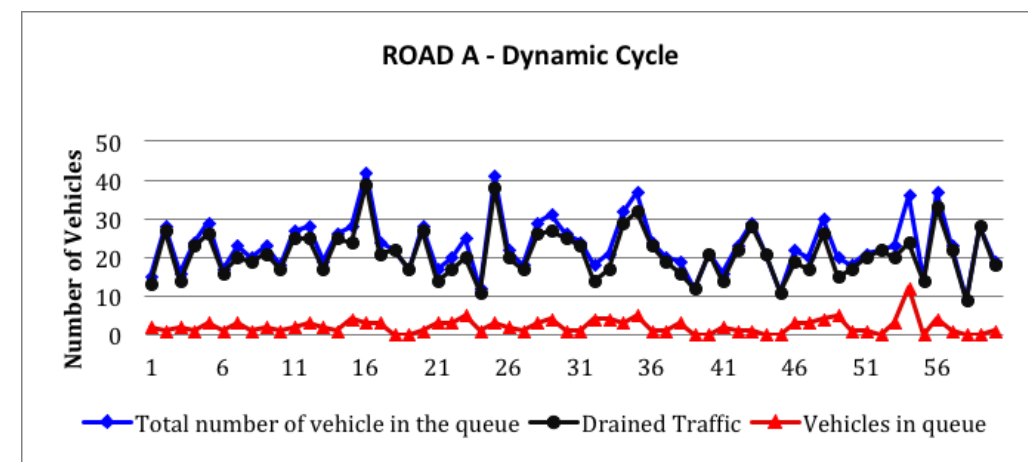

Figure 8. Road A - measures obtained with fixed-cycle

The Figure 9 shows measurements carried out on road B in case of fixed-cycle. In this case, sensors detect on average 21.4 vehicles/min in transit. Of these, just 10.53 are disposed.

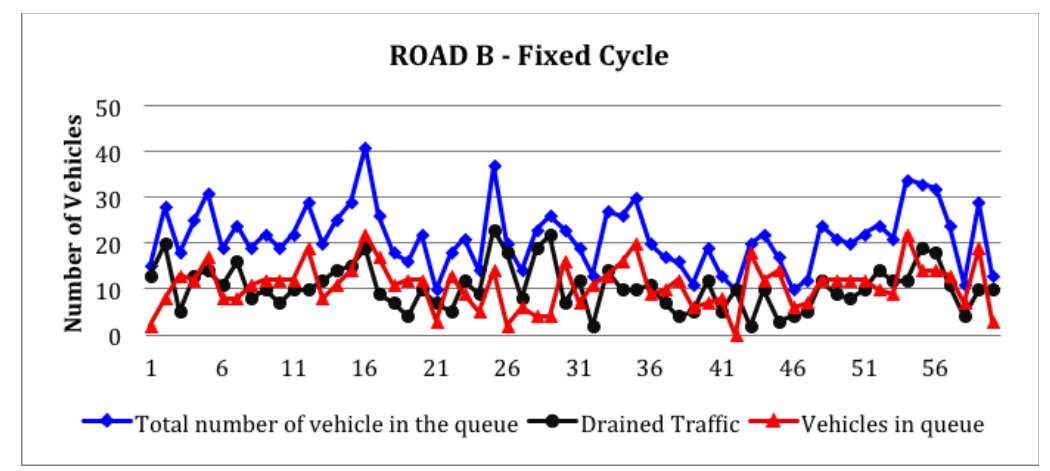

Figure 9. Road B - measures obtained with dynamic-cycle

In case of dynamic-cycle, as shown on Figure 10, on average 10.6 vehicle/minute are drained (about $87 \%$ respect to the total number of vehicles transited).



Figure 10. Road B - measures obtained with dynamic-cycle

\subsection{Numerical simulations}

Two different simulations have been conducted to evaluate the response variation, both in terms of electric potential and electric power generated by electromechanical coupling. The analysis has been conducted through the development of some 3D numerical models using the FEM code "COMSOL MULTIPHYSICS 4.2" [33] and "QUCS 0.0.16"[34] for the electromechanic coupling and the simulation respectively. The FEM model consists of a free tetrahedric mesh made by 14.658 solid elements (90.514 degree 
of freedom). Firstly, a static analysis was made by applying a displacement imposed to the upper surface of the box, of $10 \mathrm{~mm}$ (Figure 6). Then, two dynamical studies were made with and without the circuit respectively. The numerical simulation was performed taking in considerations two different piezo's polarization configurations, [35] with the main aim to evaluate how the model's response changes by changing the polarization direction and resistive load applied [26]. The simulation results show how the increasing of the R2 resistance value (from $100 \mathrm{ohm}$ to $20.000 \mathrm{ohm}$ ) produces a more stable load voltage over the time, both for the serial and the parallel configuration (Figure 11). More in detail, in the serial configuration the load voltage varies from $0,0 \mathrm{~V}$ to $10,0 \mathrm{~V}$ while in the parallel configuration values varies from $0,0 \mathrm{~V}$ to $6,0 \mathrm{~V}$ as shown on Figure 12.

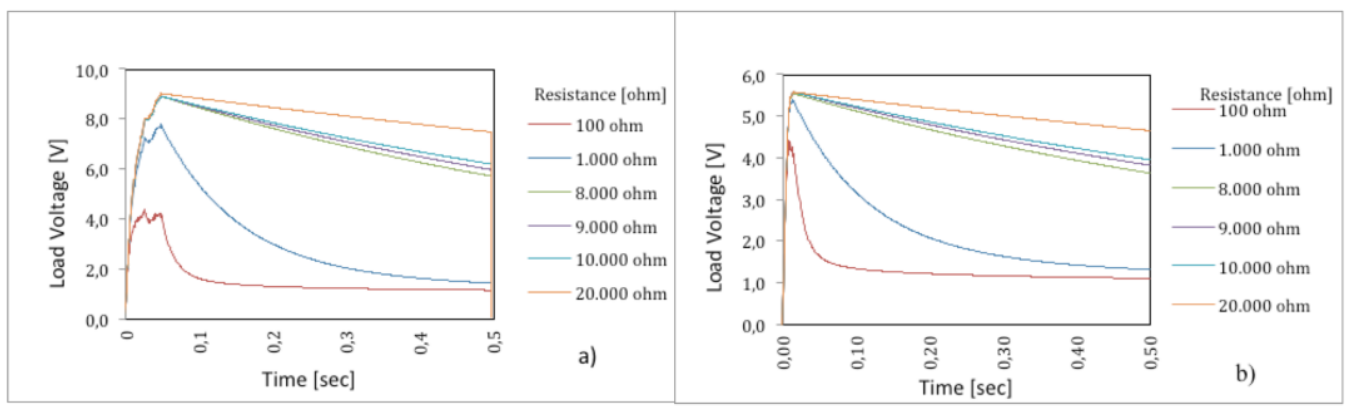

Figure 11. Load Voltage vs Time plotting for different resistance R2 values: a) Serial configuration;

b) Parallel configuration

By comparing the peak voltages measured using the parallel and serial polarization respectively as shown in Table 2, it is possible to verify how the serial configuration produces little better performance than the parallel configuration as shown on Figure 12.

Table 2. Average voltage and power harvested from the system vs. load resistance

\begin{tabular}{|c|c|c|c|c|c|c|c|c|}
\hline \multirow{3}{*}{$\begin{array}{c}\text { Serial } \\
\text { Configuration }\end{array}$} & $\begin{array}{c}\mathbf{R} \\
\text { load }\end{array}$ & {$[\Omega]$} & $1,00 \mathrm{E}+02$ & $1,00 \mathrm{E}+03$ & $8,00 \mathrm{E}+03$ & $9,00 \mathrm{E}+03$ & $1,00 \mathrm{E}+04$ & $2,00 \mathrm{E}+04$ \\
\hline & $\overline{\mathrm{Vm}}$ & [V] & 0,25 & 2,20 & 5,80 & 6,01 & 6,20 & 7,03 \\
\hline & $\mathbf{P}$ & {$[\mathrm{mW}]$} & 0,65 & 4,84 & 4,21 & 4,01 & 3,84 & 2,47 \\
\hline \multirow{3}{*}{$\begin{array}{c}\text { Parallel } \\
\text { Configuration }\end{array}$} & $\begin{array}{c}\mathbf{R} \\
\text { load }\end{array}$ & {$[\Omega]$} & $1,00 \mathrm{E}+02$ & $1,00 \mathrm{E}+03$ & $8,00 \mathrm{E}+03$ & $9,00 \mathrm{E}+03$ & $1,00 \mathrm{E}+04$ & $2,00 \mathrm{E}+04$ \\
\hline & Vm & [V] & 0,35 & 2,25 & 4,49 & 4,66 & 5,05 & 5,45 \\
\hline & $\mathbf{P}$ & {$[\mathrm{mW}]$} & 1,13 & 5,06 & 2,52 & 2,33 & 2,17 & 1,28 \\
\hline
\end{tabular}

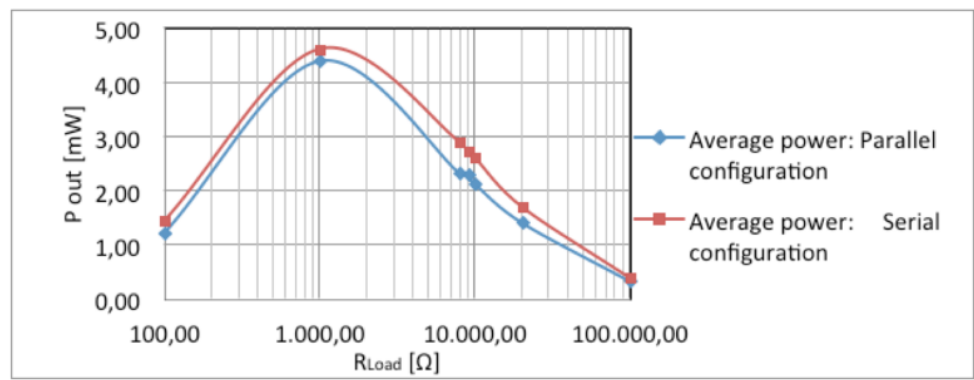

Figure 12.Comparison between the average electrical powers harvested from the system depending from the load Resistance

\section{Conclusions}

The wireless sensor networks are used in several applications, as mentioned above, for several features. The possibility to use real-time information to dynamically manipulate the traffic light cycles is a key aspect of intelligent transportation systems. At the same time, however, the sensor devices are battery powered and, after their normal duration (dependent on the use) must be replaced. In this paper 
a novel technique for the dynamic management of traffic lights to reduce queues has been presented. This technique is based on information provided by a wireless sensor network powered by energy harvesting devices inserted within a road cavity in order to catch the external vibrations, caused by the passage of vehicles. Obtained results are very promising both in terms of queues management and in terms of the proposed energy harvesting technique. The numerical simulation of the system was performed by applying the 3D numerical modelling techniques through the use of the F.E.M. code "Comsol Multiphysics 4.2", while for the electromechanical coupling and the simulation the program "QUCS 0.0.16" has been used. The positive results obtained from the numerical analysis above, led to experimental applications.

\section{References}

1. Collotta, M., Pau, G., Salerno, V. M., Scata, G. (2012). A Novel Road Monitoring Approach Using Wireless Sensor Networks. In The Sixth International Conference on Complex, Intelligent and Software Intensive Systems (CISIS), July 4-6, 2012 (pp. 376-381). Palermo, ITALY.

2. Hao Wu, Lihua Tang, Yaowen Yang, Chee Kiong Soh (2013). A novel two-degrees-of-freedom piezoelectric energy harvester. Journal of Intelligent Material Systems and Structures, 24, 357.

3. Yang, R., Qin, R., Li, C., Zhu, G., Wang, Z. L. (2009). Converting biomechanical energy into electricity by a muscle-movement-driven nanogenerator. Nano Lett., 9, 1201-1205.

4. Chopra I. (2002). Review of State-of-Art of Smart Structures and Integrated Systems. AIAA Journal, 40, 2145-2187.

5. Roundy, S., Wright, P.K., Rabaey, J. (2003). Energy scavenging for wireless sensor networks with special focus on vibrations. Kluwer Academic Press.

6. Vitabile, S., Conti, V., Collotta, M., Scatà, G., Andolina, S., Gentile, A., Sorbello, F. (2012). A real-time network architecture for biometric data delivery in Ambient Intelligence. Journal of Ambient Intelligence and Humanized Computing, 1-19.

7. Collotta, M., Nicolosi, G., Toscano, E., Mirabella, O. (2008). A ZigBee-based network for home heating control. In The 34th Annual Conference of IEEE Industrial Electronics (IECON), November 10-13, 2008 (pp. 2724-2729). Orlando (FL), USA.

8. Changzhan, Gu, Rice, J. A., Changzhi, Li (2012). A wireless smart sensor network based on multifunction interferometric radar sensors for structural health monitoring. In IEEE Topical Conference on Wireless Sensors and Sensor Networks (WiSNet), January 15-18 (pp. 33-36). Santa Clara (CA), USA.

9. Collotta, M., Conti, V., Pau, G., Scatà, G., Vitabile, S. (2012). Fuzzy Techniques for Access and Data Management in Home Automation Environments. Journal of Mobile Multimedia, 8(3), 181-203.

10. Collotta, M., Gentile, L., Pau, G., Scata, G. (2012). A Dynamic Algorithm to Improve Industrial Wireless Sensor Networks Management. In The 38th Annual Conference of IEEE Industrial Electronics (IECON), October 25-28, 2012 (pp. 2802-2807). Montreal, Canada.

11. Collotta, M., Lo Bello, L., Toscano, E. (2009). A Proposal towards flexible wireless communication in factory automation based on the IEEE 802.15.4 protocol. In IEEE Conference on Emerging Technologies \& Factory Automation (ETFA), September 22-25, 2009 (pp. 1-4). Mallorca, Spain.

12. Xiao Laisheng, Peng Xiaohong, Wang Zhengxia (2009). Research on Traffic Monitoring Network and its Traffic Flow Forecast and Congestion Control Model Based on Wireless Sensor Network. In International Conference on Measuring Technology and Mechatronics Automation (ICMTMA), April 11-12, 2009 (pp. 142-147). Zhangjiajie (Hunan), China.

13. Ali Ahammed, G.F., Banu, R. (2010). Analyzing the Performance of Active Queue Management Algorithms. International Journal of Computer Networks \& Communications, 2(2).

14. Tubaishat, M., Yi Shang, Hongchi Shi. (2007). Adaptive Traffic Light Control with Wireless Sensor Networks. In The $4^{\text {th }}$ IEEE Consumer Communications and Networking Conference (CCNC), January 2007 (pp. 187-191). Las Vegas (NV), USA.

15. Binbin Zhou, Jiannong Cao, Hejun Wu (2011). Adaptive Traffic Light Control of Multiple Intersections in WSN-based ITS. In IEEE 73rd Vehicular Technology Conference - VTC Spring, May 15-18 (pp. 1-5). Yokohama, Japan.

16. Binbin Zhou, Jiannong Cao, Xiaoqin Zeng, Hejun Wu (2010). Adaptive Traffic Light Control in Wireless Sensor Network-based Intelligent Transportation System. In IEEE 72nd Vehicular Technology Conference Fall - VTC 2010-Fall, September 6-9, 2010 (pp. 1-5). Ottawa (ON), Canada.

17. Senanayake, S., M., R.,Divarathne, H., K., D., W., M., M., R.,Samaranayake, B. G. L. T. (2009). Realtime dynamic traffic light timing adaption algorithm and simulation software. In International Conference on Industrial and Information Systems (ICIIS), December 28-31, 2009 (pp. 536-567). Sri Lanka.

18. Fuqiang Zou, Yang, B., Yitao Cao (2009). Traffic light control for a single intersection based on wireless sensor network. In The 9th International Conference on Electronic Measurement \& Instruments (ICEMI), 
August 16-19, 2009 (pp. 1040-1044). Beijing-China.

19. Messineo, A., Panno, G. (2011). LNG cold energy use in agro-food industry: A case study in Sicily. Journal of Natural Gas Science and Engineering, 3, 356-363.

20. Panno, D., Messineo, A., Dispenza, A. (2007). Cogeneration plant in a pasta factory, energy saving and environmental benefit. Energy, 32, 746-754.

21. Messineo, A., Marchese, F. (2008). Performance evaluation of hybrid RO/MEE systems powered by a WTE plant. Desalination, 229, 82-93.

22. Abramovich, H., Milgrom, C., Harash, E., Azulay, L., E., Amit, U. (2010) Patent application title: Multilayer modular energy harvesting apparatus, system and method. INNOWATTECH LTD., 02/25/2010.

23. Messineo, A., Alaimo, A., Denaro, M., Ticali, D. (2012). Piezoelectric bender transducers for energy harvesting applications. Energy Procedia, 14, 39-44. Doi: 10.1016/j.egypro.2011.12.894.

24. IEEE Standard for Information Technology. (June 2006). Wireless Medium Access Control (MAC) and Physical Layer (PHY) Specifications for Low-Rate Wireless Personal Area Networks (LR- WPANs)", Part 15.4. 802.15.4.

25. Collotta, M., Pau, G., Salerno, V.M., Scata, G. (2011). A fuzzy based algorithm to manage power consumption in industrial Wireless Sensor Networks. In The 9th IEEE International Conference on Industrial Informatics (INDIN), July 26-29, 2011 (pp. 151-156). Lisbon, Portugal.

26. Ticali, D., Denaro, M., Barracco, A., Guarrieri, M. (2012). Electro Mechanical Characterization of a Bimorph Piezo for Energy Harvesting Applications in Road Infrastructures. European Journal of Scientific Research, 90(2), 212-217.

27. Mitcheson, P. D., Green, T. C., Yeatman, E. M., Holmes, A. S. (2004). Architectures for VibrationDriven Micropower Generators. Journal of micro-electromechanical system, 13(3).

28. Scholer, C., Ikeler, J., Ramirez, J., Jen, S., Falcon, G. (2009). Piezoelectric Harvesting. A sustainable approach to clean energy generation in airport terminal. April 13, 2009.

29. Sodano, H.A., Inman, D.J., Park, G. (2005). Comparison of Piezoelectric Energy Harvesting devices for recharging batteries. Journal of Intelligent Material System and Structures, 16.

30. Guilar, N. J., Rajeevan Amirtharajah, Hurst, P. J. (2009). A Full Wave Rectifier With Integrated Peak Selection for Multiple Electrode Piezoelectric Energy Harvesters. IEEE Journal of Solid State Circuits, 44(1), 240-246.

31. Lallart, M., Lauric G., Richard, C., Guyomar, D. (2008). Double Synchronized Switch Harvesting (DSSH): A New Energy Harvesting Scheme for Efficient Energy Extraction. IEEE Transaction on Ultrasonic, Ferroelectric and Frequency Control, 55(10), 2119-2130.

32. Guyomar, D., Lallart, M (2011). Recent Progress in Piezoelectric Conversion and Energy Harvesting Using Nonlinear Electronic Interfaces and Issues in Small Scale Implementation. Journal Micromachines, 2, 274-294.

33. COMSOL 4.2. User manual, COMSOL MULTIPHYSICS.

34. Qucs (undated). Qucs project. http://qucs.sourceforge.net/

35. Leland, E. S., Baker, J., Carleton, E., Reilly, E., Lai, E., Otis, B, Rabaey, J. M., Wright, P. K. (2005). Improving Power output for vibration based energy scavengers. IEEE Pervasive Computing, 4(1), 28-36. 